\title{
Sepsis neonatal temprana, incidencia y factores de riesgo asociados en un hospital público del occidente de México
}

\author{
René Oswaldo Pérez, Juan Carlos Lona, Moisés Quiles, Miguel Ángel Verdugo, \\ Elba Patricia Ascencio y Edith Adriana Benítez
}

\section{Early neonatal sepsis, incidence and associated risk factors in a public hospital in western Mexico}

Background: Early neonatal sepsis (EOS) is the second leading cause of death in the first week of life. Epidemiology differs in developed and developing countries. Aim: To describe the epidemiology of EOS among newborn patients in a public hospital in western Mexico. Methods: A prospective cohort study was performed in newborns of Nuevo Hospital Civil de Guadalajara "Dr. Juan I Menchaca". EOS was diagnosed with blood cultures or cultures of cerebrospinal fluid within the first $72 \mathrm{~h}$ of life. We analyzed risk factors (RF) by multivariate analysis with logistic regression. Results: We identified an EOS incidence of 4.7 events per 1,000 live births. Seventy two percent of the isolated bacteria were gram negative bacilli. Factors associated with EOS were maternal age $\leq 15$ years (OR 3.50; 95\% CI 1.56-7.85), rupture of membranes > $18 \mathrm{~h}$ (OR 2.65; 95\% CI 1.18-5.92), maternal fever (OR 6.04; 95\% CI 1.54-23.6), birth weight $\leq 2,500 \mathrm{~g}(\mathrm{OR} 4.82 ; 95 \%$ CI 2.38-9.75) and gestational age $<37$ weeks (OR 3.14; 95\% CI 1.58-6.22). Conclusions: In addition to the RF known for EOS an independent association was observed with maternal age $\leq 15$ years.

Key words: Sepsis, risk factors, adolescent pregnancy, bacteremia, meningitis.

Palabras clave: Sepsis, factores de riesgo, embarazo adolescente, bacteriemia, meningitis.

\section{Introducción}

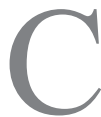
ada año se presentan cuatro millones de muertes neonatales en el mundo y $35 \%$ son debidas a procesos infecciosos ${ }^{1-3}$. La sepsis neonatal temprana (SNT) se define como una infección demostrada en sangre o en líquido cefalorraquídeo (LCR) en pacientes con 72 h de vida o menos ${ }^{2}$. En Estados Unidos de América (E.U.A.) la incidencia de SNT se estima en 1 a 2 casos por 1.000 nacidos vivos ${ }^{2,4}$; sin embargo, en países en vías de desarrollo varía de 2,2 a 9,8 eventos por 1.000 nacidos vivos $^{1}$. En México, la sepsis bacteriana del recién nacido (RN) es la segunda causa de muerte (12,3\%), en pacientes de uno a seis días de vida ${ }^{5}$.

Los RN presentan mayor riesgo de infecciones debido, entre otras condiciones, a su inmadurez inmunológica ${ }^{6}$. Se ha observado que los pacientes más afectados por SNT son los RN prematuros o de muy bajo peso al nacimiento, principalmente los que son expuestos a infecciones maternas o con disrupción de las membranas amnióticas ${ }^{2,7-12}$. Streptococcus agalactiae es el principal microorganismo aislado en estas infecciones; sin embargo, en países en vías de desarrollo las bacterias predominantes son enterobacterias. Las razones de estas diferencias no son claramente entendidas ${ }^{1}$.

El estándar de oro para el diagnóstico de SNT lo conforman el hemocultivo y el cultivo de $\mathrm{LCR}^{13-15}$. Los reactantes de fase aguda como la proteína $\mathrm{C}$ reactiva (PCR) han evidenciado utilidad como pruebas de tamizaje por su alto valor predictor negativo ${ }^{14,16}$. Las pruebas diagnósticas moleculares no han mostrado mayor efectividad que los estudios microbiológicos ${ }^{13}$ pero es probable que en pacientes con antecedente de exposición a antimicrobianos su utilidad sea mayor.

Debido a que las manifestaciones clínicas de SNT son inespecíficas ${ }^{17-19}$ y que existen dificultades en la obtención oportuna de los resultados de estudios microbiológicos, la información epidemiológica permite identificar a los pacientes con mayor riesgo de sepsis y ayuda a valorar la utilidad de la aplicación universal de medidas preventivas de SNT.

Se estima que más de $95 \%$ de la información epidemiológica se origina en países desarrollados, donde los indicadores difieren de los de países en vías de desarrollo ${ }^{1}$. En el Nuevo Hospital Civil de Guadalajara "Dr. Juan I. Menchaca" (HCGJIM) no existen datos históricos en el tema. El objetivo de este estudio fue describir la epidemiología de la SNT en los RN de este hospital.

\section{Material y Métodos}

Se realizó un estudio de cohorte prospectivo, en el HCGJIM de la Ciudad de Guadalajara, Jalisco. La

\author{
Nuevo Hospital Civil de \\ Guadalajara "Dr. Juan I. \\ Menchaca", México. \\ Servicio de Neonatología (ROP, MQ) \\ Servicio de Infectología (JCL) \\ Residente Servicio de \\ Pediatría (MAV). \\ Químico farmacobiólogo (EPA) \\ Universidad de Guadalajara \\ Centro Universitario de Ciencias de \\ la Salud, Estudiante de \\ Medicina (EAB).
}

No existió financiamiento externo. Los autores declaran no presentar conflictos de interés en la realización del estudio.

Recibido: 26 de enero de 2015 Aceptado: 4 de mayo de 2015 
institución otorga servicios de salud a población abierta de escasos recursos económicos. El hospital no tiene un programa de diagnóstico materno prenatal de infección por $S$. agalactiae. El servicio de neonatología está integrado por la terapia intensiva neonatal con 18 cunas y 67 cunas de terapia intermedia.

El período de estudio fue desde el 7 de marzo de 2013 al 4 de julio de 2014. Se incluyeron en la cohorte todos los pacientes nacidos en el HCGJIM. El cálculo del tamaño de muestra se realizó con la finalidad de identificar una prevalencia de SNT de $0,1 \%$ en población infinita. El tamaño de la muestra también fue adecuado para la búsqueda de los factores de riesgo.

El diagnóstico de SNT se estableció cuando los RN hospitalizados presentaron crecimiento microbiano en cultivos de sangre o LCR obtenidos antes de las $72 \mathrm{~h}$ de vida. La toma de estudios microbiológicos fue acorde al protocolo de diagnóstico de sepsis neonatal de la institución ${ }^{20}$. Se tomaron muestras para cultivos de sangre y LCR a los RN con más de una manifestación clínica y/o pruebas de laboratorio anormales (fiebre, hipotermia, taquicardia, bradicardia, polipnea, leucocitosis, leucopenia o PCR > $1,0 \mathrm{mg} / \mathrm{dl}$ ) y a los $\mathrm{RN}$ con cualquier manifestación clínica y uno o más de los siguientes antecedentes maternos: infección de vías urinarias activa, corioamnionitis, fiebre y ruptura prematura de membranas $>18 \mathrm{~h}$.

Manifestaciones clínicas consideradas como sugestivas de sepsis fueron hipoactividad, dificultad para la alimentación (hiporexia, vómito o diarrea), dificultad respiratoria y cianosis ${ }^{17,20}$.

\section{Definiciones}

Leucocitosis: Recuento de leucocitos séricos $>34 \mathrm{x}$ $10^{3} / \mathrm{mm}^{3}$. Leucopenia: recuento leucocitario $<5 \times 10^{3} /$ $\mathrm{mm}^{3}$. Fiebre: temperatura cutánea $\geq 37,5^{\circ} \mathrm{C}$. Hipotermia $<35,5^{\circ} \mathrm{C}$. Bradicardia: frecuencia cardíaca $(\mathrm{FC})<100$ latidos/min. Taquicardia: FC $>180$ latidos/min. Los valores de PCR $>1,0 \mathrm{mg} / \mathrm{dl}$ se clasificaron como anormales.

Para los hemocultivos se obtuvieron dos o más muestras de sangre por punción de venas periféricas en sitios diferentes, con técnica aséptica ${ }^{21}$ y se inocularon en frascos de hemocultivo (BacT/ALERT PF Pediatric FAN $^{\circledR}$ ). Se vigiló el crecimiento microbiano en sistema automatizado Bact/ALERT ${ }^{\circledR}$ 3D durante 7 días. Los cultivos detectados como positivos fueron resembrados en agar sangre y McConkey. La prueba se consideró positiva si existió crecimiento de bacterias gramnegativas o levaduras en uno o más frascos. Para bacterias grampositivas se clasificaron como cultivos positivos si el aislamiento se realizó en dos o más frascos; si sólo se aislaron de un frasco se catalogaron como contaminantes ${ }^{15,21,22}$.

La obtención de LCR se realizó mediante punción lumbar con técnica estéril. La muestra se inoculó en medios enriquecidos (BacT/ALERT PF Pediatric FAN ${ }^{\circledR}$ ) y en agar sangre. Cualquier crecimiento bacteriano se consideró significativo excepto el hallazgo de Staphylococcus coagulasa negativa; en estos casos se clasificó como evento infeccioso, sólo si existió anormalidad en el número de leucocitos y glucosa del estudio citoquímico de LCR.

En las bacterias aisladas en cada evento de SNT se identificó género y especie por el sistema automatizado MicroScan autoSCAN-4 System ${ }^{\circledR}$.

De los expedientes clínicos de los RN se obtuvo información sobre factores de riesgo para SNT, ya definidos en la literatura científica:2,7-11 número de consultas en control prenatal, vía de nacimiento, género del RN, edad gestacional estimada por escala de Capurro o Ballard, Apgar al minuto de vida, peso al nacimiento y factores de riesgo maternos (ruptura de membranas $>18 \mathrm{~h}$, infección de vías urinarias, corio-amnionitis y fiebre materna); también se registró la edad materna.

\section{Análisis estadístico}

Se estimó la incidencia de SNT en el total de pacientes RN y posteriormente para subgrupos, en función de la presencia o ausencia de los factores estudiados.

De las variables cualitativas se cuantificaron frecuencias y porcentajes. La búsqueda de asociación con la variable dependiente se evalúo con riesgo relativo e intervalos de confianza a $95 \%$ en programa open-epi (http://www.openepi.com/Menu/OE Menu.htm). De las variables cuantitativas se calculó media, desviación estándar (DE) y rangos. La búsqueda de asociación con la variable dependiente se realizó con análisis bivariado con prueba t de Student. Para las variables peso, edad gestacional, edad materna y control prenatal se generaron variables dicotómicas con diferentes puntos de corte. Las variables que presentaron valor de $p$ menor a 0,2 se incluyeron en análisis multivariado con regresión logística en programa IBM SPSS Statistics Versión 20.

El proyecto fue aprobado por los Comités de Ética e Investigación del HCGJIM con registro \# 00073.

\section{Resultados}

Durante el período de estudio se registraron 14.207 pacientes RN, 51,4\% (n: 7.298) del género masculino y 48,6\% (n: 6.907) del género femenino; en dos pacientes éste fue indeterminado por presentar genitales ambiguos.

La edad gestacional promedio fue de 38,5 semanas (máxima 42, mínima 22, DE 2,11). El 12\% (n: 1.711) presentó una edad gestacional menor a 37 semanas y $2,1 \%$ (n: 295) edad igual o menor a 32 semanas.

El peso promedio de los pacientes fue de $3.010 \mathrm{~g}$ (máximo 5.725, mínimo 440, DE 595,6). El 22,3\% (n: 3.172 ) presentó peso $\leq 2.500$ g y $2,5 \%$ (n: 360$)$ peso muy bajo al nacimiento $\leq 1.500 \mathrm{~g}$.

El promedio de edad de las madres fue de 24 años 
Tabla 1. Aislamientos microbianos en pacientes con sepsis neonatal temprana

\section{Género y especies}

Enterobacterias

Escherichia coli

Klebsiella pneumoniae

Enterobacter cloacae

Citrobacter sp

Proteus mirabilis

Yersinia enterocolitica

Shigella sp

Streptococcus

Streptococcus bovis

Streptococcus pneumoniae

Streptococcus agalactiae

Streptococcus pyogenes

Enterococcus

Enterococcus faecalis

Enterococcus faecium

Staphylococcus

Staphylococcus aureus

Staphylococcus coagulasa negativa

Bacterias no fermentadoras

Pseudomonas sp

Acinetobacter $\mathrm{sp}$

Flavobacterium sp

El número de aislamientos microbianos excede el número de eventos de sepsis neonatal por un cultivo poli-microbiano.

(máxima 48, mínima 12, DE 6,48). El 4,8\% (n: 680) refirió antecedente de control prenatal nulo y $23,7 \%$ (n: 3.365) acudió a menos de cinco consultas de evaluación antes del parto. El 59,5\% (n: 8.452) de los nacimientos fue por vía vaginal y el resto por cesárea 40,5\% (n: 5.755).

Se hospitalizó 10,9\% (n: 1.550) de los RN y se realizó escrutinio diagnóstico para SNT en 4,2\% (n: 602). Se cuantificaron 67 eventos de SNT, la incidencia fue de 4,7 eventos por $1.000 \mathrm{RN}$ vivos (IC 95\% 3,7-5,9).

En 94\% (n: 63) se obtuvo aislamiento microbiano en el torrente sanguíneo, uno en LCR y en tres casos se recuperó de ambos sitios. El 72\% de las bacterias identificadas fueron bacilos gramnegativos, las más frecuentes Escherichia coli y Klebsiella pneumoniae.

Los pacientes con infección por bacterias gramnegativas tuvieron menor peso y edad gestacional $(2.028 \mathrm{~g} / 35,1$ semanas) que los pacientes con infección por bacterias grampositivas $(2.562 \mathrm{~g} / 36,9$ semanas) (p 0,02; p 0,04). Al comparar los pacientes con infección por $E$. coli y $K$. pneumoniae no se encontraron diferencias significativas. El total de bacterias identificadas se muestra en la Tabla 1.

La incidencia de SNT en función de la presencia o ausencia de factores de riesgo y el riesgo relativo con intervalos de confianza al $95 \%$ se muestran en la Tabla 2.
Tabla 2. Incidencia de sepsis neonatal temprana en función de factores de riesgo con riesgo relativo e IC al $95 \%$

$\begin{array}{cccccc}\mathrm{n} \text { de } & \text { Eventos } & \begin{array}{c}\text { Incidencia/ } \\ \text { pacientes }\end{array} & \text { de SNT } & \begin{array}{c}1.000 \\ \text { pacientes }\end{array} & \end{array}$

Género

$\begin{array}{lllllll}\text { Femenino } & 6.907 & 34 & 4,9 & 1,1 & 0,67-1,7 & 0,72 \\ \text { Masculino } & 7.298 & 33 & 4,5 & & & \\ \begin{array}{l}\text { Vía de nacimiento } \\ \text { Cesárea }\end{array} & 5.755 & 34 & 5,9 & 1,5 & 0,94-2,4 & 0,08 \\ \text { Parto } & 8.452 & 33 & 3,9 & & & \end{array}$

Control prenatal nulo

$\begin{array}{lllllll}\text { Presente } & 680 & 3 & 4,4 & 0,93 & 0,29-2,96 & 0,90\end{array}$

Ausente

$13.527 \quad 64 \quad 4,7$

Control prenatal $<5$ consultas

Presente

$3.365 \quad 26$

Ausente

10.842

26

7,7

$2,04 \quad 1,25-3,33$

0,003

Madre $\leq 15$ años

Ausente

442

41

3,8

Ruptura de membranas $>18 \mathrm{~h}$

Presente

13.765

7

15,8

3,6

$1,67-7,90<0,001$

Ausente

132

60

4,35

Corioamnonitis materna

Presente

Ausente

14.075

$$
9
$$

68,1

$16,55 \quad 8,37-32,7<0,001$

nfección urinaria materna

Presente

19

58

4,12

Ausente

14.188

3

157,8

7,8
4,51

35

$12-101,7<0,001$

Fiebre materna

Presente

Ausente

14.184

64

50

Apgar al minuto $\leq 7$

Presente

1.883

Ausente

12324

4.108

$$
5
$$

50,5

11,49

$4,7-27,9$

4,4

Peso $\leq 2.500 \mathrm{~g}$

Presente

Ausente

2158

62

4,4

$\sqrt{2}$

$<$

Peso $\leq 1.500 \mathrm{~g}$

Presente

12049

3

130,4

$28,91 \quad 9,79-85,4<0,001$

\section{Ausente}

360

13847

64

4,5

Edad gestacional $<37$ semanas

Presente

1711

Ausente

12496

33

17,5

6

Edad gestacional $\leq 32$ semanas

Presente

295

13912
2,75

34

45

22

20,8

11,4

1,82
$6,87-18,9<0,001$

\section{7}

47,2

13,08

$7,6-22,4<0,001$

3,61

40

23,4

$10,826,66-17,58<0,001$

27

2,16

$\begin{array}{lrrr}11 & 37,2 & 9,26 & 4,9-17,5<0,001 \\ 56 & 4,0 & \end{array}$


Tabla 3. Análisis multivariado (regresión logística) de factores asociados a sepsis neonatal temprana

\begin{tabular}{lccc} 
& $\begin{array}{c}\text { Exp (B) } \\
\text { OR }\end{array}$ & \multicolumn{2}{c}{ IC al 95\% para OR } \\
Inferior & Superior \\
Madre $\leq 15$ años & 3,50 & 1,56 & 7,85 \\
Ruptura de membranas $>18 \mathrm{~h}$ & 2,65 & 1,18 & 5,92 \\
Fiebre materna & 6,04 & 1,54 & 23,6 \\
Peso $\leq 2.500 \mathrm{~g}$ & 4,82 & 2,38 & 9,75 \\
Edad gestacional $<37$ semanas & 3,14 & 1,58 & 6,22 \\
OR: odds ratio; IC: intervalos de confianza. & &
\end{tabular}

Las variables que presentaron valor de $\mathrm{p}<0,2$ se incluyeron en análisis multivariado con regresión logística. Para obtener el modelo final se introdujo el total de las variables que cumplían el criterio y se excluyeron, paso a paso, en función de su significancia estadística o la influencia que tenían para otras variables dentro del modelo. Los factores de riesgo identificados mediante análisis multivariado se enlistan en la Tabla 3.

La letalidad de SNT fue de 9\% (n: 6). Se aisló bacilos gramnegativos en cinco de las defunciones; no se observó diferencia en la letalidad de las infecciones causadas por bacterias grampositivas $(5,3 \%)$ o gramnegativas $(12,5 \%)$, (p 0,38). En pacientes con SNT, el peso $\leq 1.500 \mathrm{~g}(\mathrm{OR} 3,9$; IC $95 \% 1-15,8)$ y la edad gestacional $\leq 32$ semanas (OR 3,8 ; IC 95\% 1-14,7) se asociaron a mayor probabilidad de muerte.

\section{Discusión}

En este estudio, la incidencia de SNT (4,7 eventos por $1.000 \mathrm{RN}$ ) fue similar a la reportada por otras investigaciones en México $^{23}$; sin embargo, Leal y cols., cuantificaron en el sureste del país una incidencia de SNT de 32,8 eventos por $1.000 \mathrm{RN}$ vivos. Es posible que la diferencia se debió al uso de distintos criterios en la definición de caso ${ }^{24}$.

En países desarrollados, la causa más frecuente de SNT es $S$. agalactiae ${ }^{2}$, mientras que en países en vías de desarrollo son enterobacterias ${ }^{25-27}$. En esta investigación, 63,2\% de los eventos de SNT fueron causados por enterobacterias. Escherichia coli y K. pneumoniae fueron las bacterias aisladas con mayor frecuencia (n: 31), S. agalactiae representó 2,9\% (n: 2) de las bacterias identificadas.

En el HCGJIM no existe un programa para la detección y tratamiento de colonización materna por $S$. agalactiae; sin embargo, la incidencia de SNT causada por esta bacteria fue de 0,14 eventos por cada $1.000 \mathrm{RN}$, frecuencia menor a la reportada en E.U.A. (0,34 eventos por cada
$1.000 \mathrm{RN}$ ), donde se realizan estrategias diagnósticas y profilaxis antimicrobiana ${ }^{28,29}$.

En México, la prevalencia de colonización por $S$. agalactiae en mujeres embarazadas es de $8,6 \%$ (IC 95\% $6,8-10,5)^{30}$, mientras que en E.U.A. es de $24,2 \%{ }^{28}$; estos indicadores posiblemente influyen sobre las diferencias en la etiología de SNT, pero es conveniente indagar la contribución de otros factores como el uso de antimicrobianos prenatales aun sin pruebas microbiológicas positivas y sus consecuencias sobre los resultados en las pruebas maternas y de los RN.

Las tendencias epidemiológicas actuales han mostrado un decremento en la frecuencia de SNT causada por $S$. agalactiae y se ha relacionado con la aplicación de programas de detección y tratamiento intraparto ${ }^{29}$. Contrariamente a la información publicada, Vincent Bekker y cols., observaron que en un período de 25 años, la implementación de medidas preventivas y terapéuticas para infección o colonización materna por $S$. agalactiae se asoció a un incremento en la incidencia de SNT causada por este microorganismo $(0,11$ a 0,19 eventos por $1.000 \mathrm{RN}$ vivos) $)^{31}$; estos resultados resaltan la necesidad de estudios de costo-efectividad previos a la aplicación universal de programas preventivos, principalmente en regiones de baja incidencia.

Las condiciones que determinan la etiología de las infecciones del RN están relacionadas con la inmadurez inmunológica $^{6}$, los factores de riesgo maternos ${ }^{2,8-10}$ y los factores ambientales como el uso de profilaxis antimicrobiana o pruebas de tamizaje para la identificación temprana de enfermedades infecciosas durante el embarazo ${ }^{2,4}$. En países en vías de desarrollo, es probable que el poco o nulo acceso a las intervenciones preventivas favorezca un panorama epidemiológico diferente ${ }^{25,32,33}$, lo cual resalta la importancia de conocer información procedente de países no desarrollados.

Chin-Han Tsai y cols., observaron que los eventos de SNT por $E$. coli son más comunes en pacientes prematuros y con bajo peso al nacimiento; la fiebre materna durante el parto $(\mathrm{p}<0,035)$ y la ruptura de membranas $>24 \mathrm{~h}$ $(p<0,015)$ se identificaron como factores de riesgo ${ }^{34}$. En el presente estudio, la edad gestacional $<37$ semanas, el peso al nacimiento $\leq 2.500 \mathrm{~g}$, la ruptura de membranas $>18 \mathrm{~h}$ y la fiebre materna durante el parto se asociaron a mayor riesgo de SNT. Un factor de riesgo diferente identificado en los pacientes del HCGJIM fue la edad materna $\leq 15$ años.

Es probable que la edad materna $\leq 15$ años esté relacionada con diferentes patrones de colonización bacteriana del tracto genitourinario. En condiciones normales, la microbiota vaginal está compuesta predominantemente por Lactobacillus spp. Esta colonización es un mecanismo de protección contra procesos infecciosos por la reducción del pH vaginal y la producción de sustancias antimicrobia- 
nas como peróxido de hidrógeno. La edad de las mujeres, las modificaciones hormonales y el uso de medicamentos pueden alterar el ecosistema vaginal y causar colonización por bacterias diferentes como E. coli, Enterococcus sp, Staphylococcus sp, Gardnerella sp y Mycoplasma sp. ${ }^{35,36}$. Una alta prevalencia de colonización por $S$. agalactiae se ha observado en pacientes diabéticas, en mujeres que usan tampones y en adolescentes ${ }^{37,38}$.

En este reporte, $3,1 \%$ (n: 442) de los RN fueron hijos de mujeres de 15 o menos años de vida, con un riesgo de SNT 2,5 veces mayor al resto de los pacientes. Esta información resalta la necesidad de implementar medidas preventivas del embarazo en mujeres adolescentes debido al mayor riesgo de infecciones neonatales.

Puopolo y cols., diseñaron un modelo predictor de SNT basado en condiciones que pueden identificarse previo al nacimiento; algunos de estos factores predictores fueron la presencia de fiebre materna durante el parto (OR 2,38; IC 95\% 2,05-2,77), la edad gestacional (OR 1,09; IC 95\% $1,05-1,13)$ y el tiempo de ruptura prematura de membranas (OR 3,41; IC 95\% 2,23-5,20) $)^{10}$.

Klinger y cols., de la unidad de cuidados intensivos neonatales del Children's Medical Center de Israel, reportaron una incidencia de SNT de 24,2 eventos por 1.000 $\mathrm{RN}$ en pacientes con muy bajo peso al nacimiento. El 55\% de los patógenos aislados fueron bacilos gramnegativos y los factores asociados a la infección fueron la ausencia de control prenatal, ruptura de membranas $>24 \mathrm{~h}$ y la presencia de amnionitis ${ }^{39}$. En el HCGJIM, 2,5\% de los RN presentaron peso al nacimiento $\leq 1.500 \mathrm{~g}$ y la incidencia de SNT para este grupo de pacientes fue de 47,2 eventos por cada $1.000 \mathrm{RN}$, frecuencia significativamente mayor a la del total de la población (RR 13,08; IC 95\% 7,6-22,4).

Una limitante de este estudio es el haberse realizado con una muestra hospitalaria que puede ser diferente a la población de la cual procede; sin embargo, debido a que el HCGJIM atiende a población abierta y heterogénea las diferencias pueden ser menores.

Aunque el hemocultivo es la prueba de referencia para el diagnóstico de SNT, pueden presentarse resulta- dos falsos positivos y falsos negativos que dificultan la comparación de los resultados entre diferentes estudios ${ }^{40}$.

\section{Conclusiones}

La incidencia de SNT y agentes bacterianos aislados con mayor frecuencia fueron similares a lo reportado en estudios realizados en países en vías de desarrollo. Mediante análisis multivariado se identificó que la edad materna $\leq 15$ años, la ruptura de membranas $>18 \mathrm{~h}$, la presencia de fiebre en la madre durante el parto, la edad gestacional $<37$ semanas y el peso al nacimiento $\leq 2.500$ $\mathrm{g}$ incrementan significativamente el riesgo de SNT.

\section{Resumen}

Introducción: La sepsis neonatal temprana (SNT) es la segunda causa de muerte en la primer semana de vida; la epidemiología difiere en países desarrollados y en vías de desarrollo. Objetivo: Describir la epidemiología de SNT en recién nacidos $(\mathrm{RN})$ de un hospital público del occidente de México. Material y Métodos: Estudio de cohorte prospectivo en RN del Nuevo Hospital Civil de Guadalajara "Dr. Juan I Menchaca”. Se diagnosticó SNT con cultivos de sangre o líquido cefalorraquídeo en las primeras $72 \mathrm{~h}$ de vida. Se indagaron factores de riesgo (FR) mediante análisis multivariado con regresión logística. Resultados: La incidencia de SNT fue de 4,7 eventos por $1.000 \mathrm{RN}$ vivos. El 72\% de las bacterias aisladas correspondió a bacilos gramnegativos. Los factores asociados a SNT fueron la edad materna $\leq 15$ años (OR 3,50 ; IC 95\% 1,56-7,85), ruptura de membranas $>18 \mathrm{~h}$ (OR 2,65; IC 95\% 1,18-5,92), fiebre materna (OR 6,04; IC $95 \% 1,54-23,6)$, peso al nacimiento $\leq 2.500 \mathrm{~g}$ (OR 4,82 ; IC 95\% 2,38-9,75) y edad gestacional $<37$ semanas (OR 3,14; IC 95\% 1,58-6,22). Conclusiones: Además de los FR ya conocidos para SNT se observó asociación independiente con edad materna $\leq 15$ años.

\section{Referencias bibliográficas}

1.- Ganatra H A, Stoll B J, Zaidi A K M. International perspective on early-onset neonatal sepsis. Clin Perinatol 2010; 37 (2): 501.

2.- Puopolo K M. Epidemiology of neonatal early-onset sepsis. Neoreviews 2008; 9 (12): e571-e9.

3.- Lawn J E, Cousens S, Zupan J. Lancet Neonatal Survival Steering Team. 4 million neonatal deaths: when? where? why? Lancet $2005 ; 365$ (9462): 891-900

4.- Phares C R, Lynfield R, Farley M M,
Mohle-Boetani J, Harrison L H, Petit S. Epidemiology of invasive group B streptococcal disease in the United States, 1999-2005. JAMA 2008; 299 (17): 2056-65.

5.- Lozano-Ascencio R, Santos-Preciado J I. Mortalidad en menores de cinco años mexicanos en 2004: hacia los objetivos del milenio. Bol Med Hosp Infant Mex 2005; 62: 406-20.

6.- Lewis D B, Wilson C B. Developmental immunology and role of host defenses in fetal and neonatal susceptibility to infection. In: Remington JS, Klein JO, eds. Infectious Disease of Fetus, Newborn, and Infants. 7th ed. Philadelphia, PA: WB Saunders; 2011 pp: 80-172.

7.- Schrag S J, Hadler J L, Arnold K E, Martell-Cleary P, Reingold A, Schuchat A. Risk factors for invasive, early-onset Escherichia coli infections in the era of widespread intrapartum antibiotic use. Pediatrics 2006; 118 (2): 570-6.

8. Oddie S, Embleton N D. Risk factors for early onset neonatal group B streptococcal sepsis: case-control study. Br Med J 2002; 325 (7359): 308.

9. Bersani I, Thomas W, Speer C P. 
Chorioamnionitis-the good or the evil for neonatal outcome? J Matern Fetal Neonatal Med 2012; 25 (S1): 12-6.

10. Puopolo K M, Draper D, Wi S, Newman T B, Zupancic J, Lieberman E, et al. Estimating the probability of neonatal early-onset infection on the basis of maternal risk factors. Pediatrics 2011; 128 (5): e1155-e1163.

11. Mukhopadhyay S, Puopolo K M. Risk assessment in neonatal early onset sepsis. Semin Perinatol 2012; 36 (6): 408-15.

12. Saltigeral-Simental P, Valenzuela-Flores A, Avendaño-Barroeta E. Agentes causales de sepsis neonatal temprana y tardía: una revisión de diez años en el "Hospital Infantil Privado". Rev Enf Infec Pediatr 2007; 20 (80): 99-105.

13. Pammi M, Flores A, Leeflang M, Versalovic J. Molecular assays in the diagnosis of neonatal sepsis: a systematic review and meta-analysis. Pediatrics 2011; 128 (4): e973-e985.

14. Polin R. Management of neonates with suspected or proven early-onset bacterial sepsis. Pediatrics 2012; 129: 1006-15.

15. Buttery J. Blood cultures in newborns and children: optimizing an everyday test. Arch Dis Child Fetal Neonatal Ed. 2002; 87 (1): F25-F28.

16. Benitz W E. Adjunct laboratory tests in the diagnosis of early-onset neonatal sepsis. Clin Perinatol 2010; 37 (2): 421-38.

17. Goldstein B, Giroir B, Randolph A. International pediatric sepsis consensus conference: Definitions for sepsis and organ dysfunction in pediatrics. Pediatr Crit Care Med 2005; 6 (1): 2-8.

18. Hofer N, Müller W, Resch B. Neonates presenting with temperature symptoms: Role in the diagnosis of early onset sepsis. Pediatr Int 2012; 54 (4): 486-90.

19. Simini F. Clinical signs that predict severe illness in children under age 2 months: a multicentre study. Commentary. Lancet 2008; 371 (9607).

20. Consejo de Salubridad General. Prevención, diagnóstico y tratamiento de sepsis y choque séptico del recién nacido en el segundo y tercer nivel de atención. México: Secretaria de Salud; noviembre 2012. (Documento en línea) Referido en enero de 2013; (107 páginas en pantalla). Disponible en: http:// www.cenetec.salud.gob.mx/descargas/gpc/ CatalogoMaestro/283_GPC_SepsisNeonatal/ SS-283-12_RER_SEPSIS_ȲYCHOQUE SxPTICO_RN.pdf.

21. Hall K K, Lyman J A. Updated review of blood culture contamination. Clin Microbiol Rev 2006; 19 (4): 788-802.

22. Beekmann S E, Diekema D J, Doern G V. Determining the clinical significance of coagulase-negative staphylococci isolated from blood cultures. Infect Control Hosp Epidemiol 2005; 26 (6): 559-66.

23. Rodríguez-Weber M A, López-Candiani C, Arredondo-García J L. Morbilidad y mortalidad por sepsis neonatal en un hospital de tercer nivel de atención. Salud Pública Mex 2003; 45 (2): 90-5.

24. Leal Y, Alverez-Nemegyei J. Risk factors and prognosis for neonatal sepsis in southeastern Mexico: analysis of a four-year historic cohort follow-up. BMC Pregnancy Childbirth 2012; 12 (1): 48.

25. Zakariya B P, Bhat V, Harish B N, Babu T A, Joseph N M. Neonatal sepsis in a tertiary care hospital in South India: bacteriological profile and antibiotic sensitivity pattern. Indian J Pediat 2011; 78 (4): 413-7.

26. Shrestha S, Shrestha N, Dongol Singh S, Shrestha N C, Shrestha R P, Madhup S K. Bacterial isolates and its antibiotic susceptibility pattern in NICU. Kathmandu Univ Med J 2013; 41 (1): 66-70.

27. Viswanathan R, Singh A K, Mukherjee S, Mukherjee R, Das P, Basu S. Aetiology and antimicrobial resistance of neonatal sepsis at a tertiary care centre in eastern India: a 3 year study. Indian J Pediat 2011; 78 (4): 409-12.

28. Centers for Disease Control and Prevention. Prevention of Perinatal Group B Streptococcal Disease Revised Guidelines from CDC, 2010. MMWR 2010; 59 ( $\mathrm{N}^{\circ} \mathrm{RR}-10$ ): 2-11.

29. Simonsen K A, Anderson-Berry A L, Delair S F, Daviesa H D. Early-onset neonatal sepsis. Clin Microbiol Rev 2014; 27 (1): 21-47.

30. Ocampo-Torres M, Sánchez-Pérez H J, Nazar-Beutelspacher A, Castro-Ramírez A E,
Cordero-Ocampo B. Factores asociados a la colonización por Streptococcus del grupo B en mujeres embarazadas de Los Altos, Chiapas. Salud Pública Mex 2000; 42: 413-21.

31. Bekker Vincent, Bijlsma M W, Beek D, Kuijpers T W, Ende A. Incidence of invasive group B streptococcal disease and pathogen genotype distribution in newborn babies in the Netherlands over 25 years: a nationwide surveillance study. Lancet Infect Dis 2014; 14 : 1083-89.

32. Li Z, Xiao Z, Li Z, Zhong Q, Zhang Y, Xu F. 116 cases of neonatal early-onset or late-onset sepsis: A single center retrospective analysis on pathogenic bacteria species distribution and antimicrobial susceptibility. Int J Clin Exp Med 2013; 6 (8): 693-9.

33. Marchant E A, Boyce G K, Sadarangani M, Lavoie P M. Neonatal sepsis due to coagulasenegative staphylococci. Clin Dev Immunol 2013; 1-10.

34. Tsai C H, Chen Y Y. Characteristics of earlyonset neonatal sepsis caused by Escherichia coli. Taiwan J Obstet Gynecol 2012; 51 (1): 26-30.

35. Darmstadt G L, Zaidi A K, Stoll B J. Neonatal infections: a global perspective. In: Remington JS, Klein JO, eds. Infectious Disease of Fetus, Newborn, and Infants. 7th ed. Philadelphia, PA: WB Saunders; 2011: 24-51.

36. Nardis C, Mosca L. Vaginal microbiota and viral sexually transmitted diseases. Ann Ig 2013; 25 (5): 443-56

37. Nandyal R R. Update on group B streptococcal infections: perinatal and neonatal periods. J Perinat Neonatal Nurs 2008; 22 (3): 230-7.

38. Persson K. Several factors influencing the colonization of group B streptococci: rectum probably the main reservoir. Scand J Infect Dis 1980; 13 (3): 171-5.

39. Klinger G, Levy I. Epidemiology and risk factors for early onset sepsis among very-lowbirthweight infants. Am J Obstet Gynecol 2009; 201 (1): 38.e1-6.

40. Wynn J L, Wong H R, Shanley T P, Bizzarro M J, Saiman L, Polin RA. Time for a neonatal-specific consensus definition for sepsis. Pediatr Crit Care Med 2014; 15: 523-8. 